\title{
Panorama Geral Sobre Doenças Inflamatórias Intestinais: Imunidade e Suscetibilidade da Doença de Crohn e Colite Ulcerativa
}

\section{Overview of Inflammatory Bowel Disease: Immunity and Susceptibility to Crohn's Disease and Ulcerative Colitis}

\author{
Francielle Bonet Ferraz ${ }^{\mathrm{a} *}$
}

\author{
${ }^{a}$ Universidade Estadual do Norte Fluminense, Programa de Pós-Graduação Stricto Sensu em Biotecnologia, RJ, Brasil \\ *E-mail: francielle.bonet@gmail.com \\ Recebido: 15 de setembro de 2015; Aceito: 15 de janeiro de 2015
}

\begin{abstract}
Resumo
Doenças Inflamatórias Intestinais (DII) são doenças autoimunes, caracterizadas por afecções sistêmicas inflamatórias crônicas, relacionadas principalmente ao trato gastrointestinal. São de cunho multifatorial, com prevalência e incidência variando de acordo com a genética, meio ambiente, microbiota intestinal e até mesmo um possível agente entérico infeccioso. Dentre as DII mais comuns, estão a Doença de Crohn e a Colite Ulcerativa, as quais possuem sintomas em comum, tais como dor abdominal, febre, diarreia e vômitos, o que pode ser confundido facilmente com gastroenterites comuns, dificultando o diagnóstico. A resposta imune às DII em atividade pode ser diferente em cada um dos tipos de acometimento do trato gastrointestinal, sendo em geral seguida por concentração elevada de citocinas pró-inflamatórias da lâmina própria da mucosa intestinal. Variações genéticas, microbiológicas e de fatores imunológicos podem ser a razão das perturbações no tecido, que contribuem para a fisiopatologia de desordens gastrointestinais. A apresentação das DII ocorre entre 15 e 25 anos ou entre 50 e 80 anos e o número de pessoas acometidas vem aumentando significativamente nas regiões Sul e Sudeste do Brasil. Há evidências de que as DII são hereditárias, no entanto, esta frequência é mais observada em pacientes com Doença de Crohn do que em pacientes com Colite Ulcerativa. Esta revisão abrange informações pertinentes sobre DII, exemplificando as mais comuns, abordando as alterações imunológicas e discutindo como o intercruzamento das duas populações mais comuns no país, no início do século XIX, foi capaz de determinar diferentes perfis genéticos e uma possível suscetibilidade às doenças.
\end{abstract}

Palavras-chave: Doenças Inflamatórias Intestinais. Doença de Crohn. Colite Ulcerativa.

\begin{abstract}
Inflammatory Bowel Diseases (IBD) are autoimmune diseases characterized by chronic inflammatory systemic diseases mainly related to the gastrointestinal tract. They are multifactorial diseases with varying prevalence and incidence according to genetics, environment, intestinal microbiota, or even an enteric infectious agent. Among the most common IBD, the Crohn's Disease and Ulcerative Colitis stand out, with symptoms such as abdominal pain, fever, diarrhea, and vomiting, which can easily be confused with common gastroenteritis, thus making diagnosis difficult. The immune response in active IBD can be different in each type of the gastrointestinal pathology, being generally followed by a high concentration of proinflammatory cytokines in the lamina propria of the intestinal mucosa. Genetic, microbiological, and immunological variations may be due to disturbances in the tissues, which contribute to the pathophysiology of gastrointestinal disorders. IBD occurs between 15-25 or 50-80 years old, and the number of people affected has increased significantly in the south and southeast of Brazil. There is evidence that IBD are inherited; however, this is most frequently observed in patients with Crohn's disease than in patients with ulcerative colitis. This review covers pertinent information about IBD, exemplifying the most common gastrointestinal pathologies, approaching immunological alterations, and discussing how the interbreeding of the two most common populations in the country in the early nineteenth century was able to determine different profiles and possible genetic susceptibility to diseases.
\end{abstract}

Keywords: Inflammatory Bowel Diseases. Crohn Disease. Colitis, Ulcerative.

\section{Introdução}

As Doenças Inflamatórias Intestinais - DII referemse a doenças autoimunes, as quais apresentam resposta imunológica inapropriada ou exacerbada e são caracterizadas por afecções sistêmicas inflamatórias crônicas, relacionadas principalmente ao trato gastrointestinal ${ }^{1}$.

As DII mais comuns são a Doença de Crohn e a Colite Ulcerativa $^{1}$, que incidem em todo o mundo e representam sério problema de saúde, uma vez que ocasionam recidivas frequentes e formas clínicas de alta gravidade $^{2}$, com repercussões importantes na qualidade de vida dos portadores ${ }^{3}$. Tanto a Doença de Crohn quanto a Colite Ulcerativa são doenças multifatoriais, o que permite considerar que a prevalência e incidência destas doenças variam de acordo com a genética, o meio ambiente, a microbiota intestinal e um possível agente entérico infeccioso ${ }^{4}$. Estes fatores podem desregular o equilíbrio entre os antígenos luminais e a resposta imune da mucosa intestinal e assim, ampliar e perpetuar a reação de inflamação ${ }^{5}$.

Devido à dificuldade de diagnóstico e tratamento, a busca de informações em artigos científicos a fim de compilar um conjunto de informações sobre o panorama geral das DII torna-se necessário, como apresentado nessa revisão, com o objetivo de auxiliar a compreensão das duas principais doenças presentes nesse grupo. 


\section{Desenvolvimento}

\subsection{Metodologia}

O presente trabalho foi desenvolvido por meio de revisão de literatura, utilizando dados bibliográficos obtidos nas bases de periódicos Capes, Lilacs, Ibecs, Medline e PubMed, SciELO e Biblioteca Digital Brasileira de Teses e Dissertações - BDTD. A estratégia de busca baseou-se nos descritores Doenças Inflamatórias Intestinais, Doença de Crohn, Colite Ulcerativa, Epidemiologia das DII, Hereditariedade das DII, e Resposta Imune Intestinal. Foram utilizadas 42 referências, no período de 1932 a 2013.

\subsection{Epidemiologia das DII}

Estudos epidemiológicos demonstram dificuldade para fornecer dados concisos, uma vez que o prognóstico da doença surge gradualmente e o diagnóstico pode ser de difícil determinação ${ }^{6}$ haja vista que sintomas como dor abdominal, febre, diarreia muco-pio-sanguinolenta, tenesmo, emagrecimento, náuseas e vômitos são compatíveis a gastroenterites comuns ${ }^{7}$. Contudo, por se tratar de doenças com componente autoimune, $25 \%$ a $40 \%$ dos pacientes apresentam sintomas extra intestinais, como complicações musculoesqueléticas, manifestações reumáticas, oculares e dermatológicas.

Sabe-se que as DII possuem uma distribuição bimodal, com a primeira ocorrência entre 15 e 25 anos e a segunda entre 50 e 80 anos, sendo a Doença de Crohn predominante no sexo feminino, possivelmente devido aos fatores hormonais que podem interferir na expressão da doença. Por outro lado, a Colite Ulcerativa parece ser discretamente mais expressa no sexo masculino 9 .

A Doença de Crohn e a Colite Ulcerativa atingem 1,4 milhão de pessoas na América do Norte ${ }^{10}$, enquanto na Europa este número é maior, atingindo 2,2 milhões de pessoas ${ }^{11}$. Ainda, estudos epidemiológicos mostram que a incidência da DII é maior nos Estados Unidos, Inglaterra, Itália, Escandinávia e nos países da região norte da Europa, tendo as menores proporções de DII na Ásia e América do Sul ${ }^{12}$ Em populações de ascendência europeia, as taxas de DII são maiores em pessoas de ascendência judaica do que em outros grupos étnicos ${ }^{13}$.

Estudos atuais demonstram um aumento significativo da incidência das DII nas regiões Sul e Sudeste do Brasil ${ }^{12}$, sugerindo, em geral, uma predominância das DII em caucasianos, o que pode estar relacionado às influências genéticas e ambientais, mas também ao estilo de vida ${ }^{14}$, como por exemplo, indivíduos com melhor padrão socioeconômico, levantando a hipótese de que possa haver associação também com higiene e saneamento básico, acarretando em uma alteração da microbiota intestinal ${ }^{15}$.

Há evidências de que as DII são hereditárias, onde 5\% a $20 \%$ dos pacientes têm histórico familiar da doença, no entanto, esta frequência é observada em geral em pacientes com Doença de Crohn quando comparada aos pacientes com Colite Ulcerativa ${ }^{16,17}$. A contribuição genética familiar é demonstrada pela taxa significativamente maior da apresentação da doença em gêmeos monozigóticos, em comparação com gêmeos dizigóticos ${ }^{16,17}$.

A prevalência, a gravidade e a resistência da doença variam consideravelmente entre os grupos étnicos, como consequência de fatores herdados e causas não determinadas ${ }^{18}$. Categorias da população, incluindo raça e grupo étnico, são claramente insuficientes para descrever completamente a variação no padrão e gama de indivíduos acometidos. Uma avaliação mais precisa da doença pode ser obtida pela genotipagem, associada a variantes genéticas em indivíduos, ao invés de utilizar apenas filiação populacional ${ }^{18}$.

\subsection{Colite Ulcerativa}

A Colite Ulcerativa é uma DII caracterizada pelo acometimento contínuo do tubo digestivo por um processo inflamatório restrito à mucosa colônica. Esta doença acomete o reto e extensões proximais variáveis do colo ${ }^{7}$, cujas alterações inflamatórias superficiais atingem à mucosa e submucosas, resultando em cripitites e abcessos nas cristas intestinais ${ }^{19}$.

Habitualmente, as alterações endoscópicas ocorrem de acordo com a severidade da doença, apresentando desde discreta presença de nódulos na mucosa, edema, hiperemia, friabilidade, perda do padrão vascular, até erosões ou úlceras recobertas por fibrina ${ }^{7}$. Tais aspectos clínicos acarretam em diarreia de intensidade moderada à severa, na maioria das vezes acompanhada de sangue vivo separado ou junto às fezes, associada à urgência evacuatória e tenesmo ${ }^{20}$.

\subsection{Doença de Crohn}

Descoberta na década de $30^{21}$, a Doença de Crohn é uma afecção inflamatória crônica de natureza transmural, segmentar, persistente e/ou recidivante ${ }^{22}$ que se localiza, principalmente, na parte distal do intestino delgado (íleo) e proximal do cólon (ceco), contudo, pode envolver qualquer segmento do trato gastrintestinal, da boca ao ânus ${ }^{22}$.

Ao contrário da Colite Ulcerativa, a Doença de Crohn demonstra a submucosa intestinal espessada, com inflamação transmural, ulcerações, fissuras e granulomas não caseosos e desse modo, apresentando complicações tais como estenose, abcessos e fístulas ${ }^{19}$. A presença de fístulas indica que a inflamação transmural penetrou nos órgãos adjacentes ou tecidos, e assim, a ativação do sistema imune leva à liberação de proteases e metaloproteinases que contribuem para a destruição dos tecidos ${ }^{7}$.

A característica mais marcante da Doença de Crohn é o acometimento transmural do trato gastrointestinal, que pode afetar todas as camadas, da mucosa à serosa e que apresenta um caráter descontínuo das lesões, devido a áreas de mucosa preservada em meio às de atividade inflamatória. Logo, podem ser observadas desde discretas erosões, edema, friabilidade, enantema, sendo mais característica a presença de úlceras, que 
podem ser aftóides, elípticas ou lineares ${ }^{23}$.

\subsection{A resposta imune nas DII}

A homeostase intestinal envolve ações coordenadas do epitélio, células do sistema imune inato e adaptativo. A permeabilidade da barreira intestinal permite a incursão microbiana, a qual é detectada por elementos do sistema imune inato, propiciando adequadas respostas tolerogênicas, inflamatórias e reconstitutivas por meio de mediadores extracelulares secretados, que recrutam outros componentes celulares, incluindo células do sistema imune adaptativo. Variações genéticas, microbiológicas e de fatores imunológicos afetam o balanço desses sinais, de modo que as perturbações no tecido contribuem para a fisiopatologia de desordens gastrointestinais, incluindo as $\mathrm{DII}^{19}$.

A barreira física do epitélio intestinal é complementada por um evoluído sistema imune inato de mucosa, que é composto por células prontas para realizar a defesa contra as incursões patogênicas e reduzir as respostas inflamatórias, a fim de manter um estado de hiporreatividade frente a bactérias comensais. As células dendríticas, macrófagos, células linfóides e neutrófilos são componentes celulares cruciais do sistema imune inato durante a infecção ou inflamação ${ }^{25}$.

Para que ocorra uma resposta humoral e celular adequadas, há necessidade da expansão clonal dos linfócitos T auxiliares, seguida pela sua ativação, que é desencadeada pela interação entre o complexo do receptor de linfócito T e a molécula CD3 com um antígeno peptídico ligado a uma molécula do $\mathrm{MHC}$ de classe II na superfície de uma célula apresentadora de antígeno. Logo, os linfócitos $\mathrm{T}$ ativados produzem citocinas que levam à ativação de outras células efetoras, que podem atuar diretamente ou produzir citocinas pró-inflamatórias e quimiocinas que, junto com a expressão das moléculas de adesão, controlam os processos de ativação e migração aos sítios inflamatórios ${ }^{26}$.

A resposta imune da mucosa gastrointestinal dos pacientes com Doença de Crohn é basicamente realizada por linfócitos T CD4 ${ }^{+}$com fenótipo Th1, caracterizado pela produção de IFN-g e IL-2, que ativam predominantemente os linfócitos $\mathrm{T}$ citotóxicos e macrófagos, os quais produzem citocinas próinflamatórias como TNF- $\alpha$ e IL- $1 \beta^{27}$.

Por outro lado, a resposta imune em pacientes com Colite Ulcerativa é realizada por linfócitos $\mathrm{T} \mathrm{CD}^{+}$com fenótipo Th2, caracterizado pela produção de TGF- $\beta$ e IL- $5^{28}$ as quais promovem o aumento da produção de IL-4, IL-5 e IL-6 que favorecem a imunidade humoral, estimulando a proliferação e diferenciação de linfócitos $\mathrm{B}$ e a consequente produção de anticorpos ${ }^{26}$.

No entanto, em geral, a concentração de citocinas próinflamatórias é elevada na lâmina própria da mucosa intestinal de pacientes com DII em atividade. Por exemplo, existe a produção espontânea de TNF- $\alpha$ por células mononucleares da lâmina própria da mucosa intestinal de pacientes com DII ativa em comparação com a mucosa normal ${ }^{28}$.
Sabe-se que citocinas pró-inflamatórias em excesso são capazes de lesar tecidos. Logo, há necessidade da modulação do processo inflamatório, que pode ser realizada pela resposta do tipo Th2, a qual é capaz de inibir paralelamente a resposta Th1, através do aumento da produção citocinas moduladoras, como IL-10, TGF- $\beta$ e IL-13 ${ }^{29}$. Mas também por IL-22, produzida por células do padrão Th17 e células $\mathrm{T} \gamma \delta$, por exemplo, cuja atividade protetora em diferentes modelos de colite demonstrou efeitos estimulantes sobre os processos antimicrobianos e reparadores ${ }^{30}$. Estudos em pacientes com Doença de Crohn mostraram frequências diminuídas de IL-22 na lâmina própria ${ }^{31}$.

A homeostase intestinal também depende do equilíbrio entre agentes oxidantes e/ou redutores tais como os radicais livres, as espécies reativas de oxigênio e os mecanismos de antioxidantes, como os que envolvem as enzimas glutationa peroxidase e glutationa S-transferase ${ }^{32}$. Este estado redox afeta muitas vias de transdução de sinais como a de NFkB e de $\mathrm{AMP}^{32}$ e, assim, contribui para sinalização intracelular, promovendo a produção de citocinas pró-inflamatórias e permitindo que haja a coordenação local da produção de quimiocinas ${ }^{33}$.

Cabe salientar que, durante a resposta inflamatória, as células do sistema imune infiltram os órgãos efetores, onde proliferam, e, com a ajuda das moléculas de adesão, migram até tecidos específicos secretando citocinas ${ }^{34}$, induzindo, desse modo, sintomas extra intestinais ${ }^{8}$.

\subsection{Genética e suscetibilidade a doenças}

A maior parte da variabilidade genética entre humanos (80\% a 90\%) ocorre dentro de populações, enquanto a menor parte da variação total é devido às diferenças entre populações ${ }^{35}$. Portanto, a maioria das populações possui alelos em comum para qualquer loco, ou seja, a presença de alelos muito frequentes pode ser observada em diversas populações distintas, como por exemplo, na população com ascendência europeia ocidental e nórdica - CEU e na de afrodescendentes da África subsaariana - YRI. Por outro lado, a pequena fração de locos que diferenciam suas frequências alélicas entre populações é suficiente para identificar as diferenças entre grupos continentais e determinar uma possível suscetibilidade à doenças ${ }^{36}$.

Devido ao comportamento imigratório que trouxe ao Brasil colônias de diversas partes do mundo, basicamente europeias, africanas, árabes e orientais, houve a diminuição do intercruzamento das duas populações mais comuns no país, as de origem africana e indígena, por meio de um artifício sócio antropológico de "europeização" da população brasileira no início do século XIX. Assim, determinando diferentes perfis genéticos ${ }^{37}$.

Pesquisas desenvolvidas no campo da genética têm demonstrado associações entre a manifestação de doenças como Fibrose Cística ${ }^{38}$, Doença Pulmonar Obstrutiva Crônica ${ }^{39}$, câncer de mama $^{40}$, Diabetes e Doença de Crohn ${ }^{41}$, com a 
ocorrência de polimorfismo de único nucleotídeo (SNP - single nucleotide polymorphism), que se refere à mudança de uma única base nitrogenada no DNA e suficiente para conduzir a apresentação da doença.

Os SNP compreendem a mais abundante forma de variação no genoma humano, com uma frequência estimada de 1 em 300 a 1 em 2000 pares de bases. No genoma humano, com aproximadamente 3,2 bilhões de pares de bases, essa frequência equivale a um número entre 1,6 e 12 milhões de SNP. Porém, os SNP têm sido estimados em cerca de 15 milhões, sendo que há cerca de 1,4 milhões já identificados ${ }^{42}$.

O impacto das diferenças de nucleotídeos é variável, mas é claramente dependente de sua localização no genoma. Embora a maioria dos SNP esteja fora das regiões codificantes dos genes, há também SNP localizados em éxons e, assim, diferenças nas regiões à montante (5') do gene podem influenciar na promoção ou repressão da transcrição gênica, resultando numa regulação diferencial da quantidade ou qualidade de proteína produzida ${ }^{19}$.

Logo, a presença de SNP em um determinado gene pode, ou não, acarretar em alterações funcionais, por isso que as relações entre polimorfismos em genes que interferem na codificação de citocinas e a resistência ou suscetibilidade a várias doenças devem ser estudadas, uma vez que tais SNP podem influenciar o curso de uma doença.

\section{Conclusão}

Obviamente que para determinar um perfil genético para as DII são necessários estudos de grandes grupos, bem caracterizados, de pacientes com riqueza de detalhes sobre sua exposição ambiental.

Cabe salientar que ainda há muito a ser aprendido sobre a etiologia das DII. Contudo, acredita-se que a hipótese da higiene é uma ferramenta importante para uma melhor compreensão de como o ambiente pode impactar intimamente a resposta imunológica da mucosa intestinal e desenvolver a doença ou induzir recidivas entre aqueles geneticamente suscetíveis à DII.

A abordagem terapêutica para os pacientes com DII restringe-se apenas para quadros sintomáticos, com o intuito de amenizar os sintomas e, possivelmente, obter um estado de remissão e/ou prevenção de recidivas. Assim, estudos mais aprofundados na área serão capazes de fornecer informações futuras mais precisas sobre a epidemiologia das DII, as quais possam gerar recomendações preventivas.

\section{Referências}

1. Bouma G, Strober W. The immunological and genetics basis of inflammatory bowel disease. Nat Rev 2003;3:521-33.

2. Jewel DP. Ulcerative colitis. In: Feldman M, Scharschmidt BF, Sleisenger MH. Sleisenger and Fordtran's gastrointestinal and liver disease: pathophysiology, diagnosis, management. Philadelphia: Saunders; 1998.

3. Pontes RMA, Mizputen SJ, Ferreira-Filho OF, Miranda C, Ferraz, MB. Qualidade de vida em pacientes portadores de doença inflamatória intestinal: tradução para o português e validação do questionário Inflammatory Bowel Disease Questionnaire (IBDQ). Arq Gastroenterol 2004;41(2):13743.

4. Hanauer SB. Inflammatory bowel disease: epidemiology, pathogenesis, and therapeutic opportunities. IBD 2006;12(1):3-9.

5. Farrel R., LeMont, J. Microbial factors in inflammatory bowel disease. Gastro Clin N Am 2002;31:41-62.

6. Victoria CR, Sassaki LY, Nunes HRC. Incidence and prevalence rates of inflammatory bowel diseases, in midwestern of São Paulo state, Brazil. Arq Gastroenterol 2009; 46(1):20-5.

7. Sands B. From symptom to diagnosis: clinical distinctions among various forms of intestinal inflammation. Gastroenterol 2004;126:1518-32.

8. Levine RM, Burakoff R. Extraintestinal manifestations of Inflammatory Bowel Disease. Gastroenterol Hepatol 2011; 7(4):235-41.

9. Figueroa CC, Quera PR, Valenzuela EJ, Jensen BC. Inflammatory bowel disease: experience of two Chilean center. Rev Med Chile 2005;304:1295-304.

10. Loftus, CG, Harmsen WS, Zinmeister, AR, Tremaine, WJ, Melton LJ, Sandborn WJ. Update on the incidence and prevalence of Crohn's disease and ulcerative colitis in Olmsted County, Minnesota, 1940-2000. IBD 2007;13(3):254-61.

11. Neu,man MG. Immune dysfunction in inflammatory bowel disease. Transl Res Rev 2007;149(4):173-86.

12. Quilici FA, Miszputen SJ. Guia prático: doença inflamatória intestinal. Rio de Janeiro: Elsevier; 2007.

13. Shugart YY, Silverberg MS, Duerr RH, Taylor KD, Wang MH, Zarfas K, et al. An SNP linkage scan identifies significant Crohn's disease loci on chromosomes 13q13.3 and, in Jewish families, on 1p35.2 and 3q29. Gen Imm 2008;9(2):161-7.

14. Wiendl H, Feger U, Mittelbronn M, Jack C, Schereiner $\mathrm{B}$, Stadelmann $\mathrm{C}$, et al. Expression of the immunetolerogenic major histocompatibility molecule HLA-G in multiple sclerosis: implications for CNS immunity. Brain 2005;128:2689-704.

15. Krishnan A, Korzenik J. Inflammatory Bowel Disease and environmental influences. Gastroenterol Clin N Am 2002;31:21-39.

16. Tysk C, Lindberg E, Jarnerot G, Flodérus-Myrhed B. Ulcerative colitis and Crohn's disease in an unselected population of monozygotic and dizygotic twins. A study of heritability and the influence of smoking. Gut 1988;39:990-6.

17. Halfvarson J. Genetics in twins with crohn's disease: less pronounced than previously believed? IBD 2011;17:6-12.

18. Rotimi CN, Lynn BJ. Ancestry and disease in the age of genomic medicine. NEJM 2010; 363:1551-58.

19. Khor B, Gardet A, Xavier RJ. Genetics and pathogenesis of inflammatory bowel disease. Nat 2011;474:307-17.

20. Judge T, Lichtenstein G. Inflammatory Bowel Disease. In: Friedman S, McQuaid K, Grendell J. Current diagnosis and treatment in gastroenterology. New York: McGraw-Hill; 2003.

21. Crohn BB, Ginzburg L, Oppenheimer GD. Regional ileitis: a pathologic and clinical entity. JAMA 1932;99:1323-29.

22. Liu C, Crawford JM. IU, C. In: Vinay K, Abbas KA, Fausto N. Patologia: bases patológicas das doenças. Rio de Janeiro: Elsevier; 2005. 
23. Machado M, Schneider N. Colonoscopia nas doenças inflamatórias intestinais. In: SOBED - Sociedade Brasileira de Endoscopia Digestiva. End Gastroenterol Terap 2006;141:995-8.

24. Souza MH, Troncon LE, Rodrigues CM, Vianna CF, Onofre $\mathrm{PH}$, Monteiro RA, et al. Trends in the occurrence (19801999) and clinical features of Crohn's disease and ulcerative colitis in an university hospital in the southeastern Brazil. Arq Gastroenterol 2002;39:98-105.

25. Blasius AL, Arnold, CN, George P, Rutschmann S, Xia Y, Lin $\mathrm{P}$, et al. Slc15a4, AP-3, and Hermansky-Pudlak syndrome proteins are required for Toll-like receptor signaling in plasmacytoid dendritic cells. PNAS 2010; 107:19973-8.

26. Sartor B. Mucosal Immunology and Mechanisms of Gastrointestinal Inflammation. In: Sleisenger M, Frieman L, Feldman M. Gastrointestinal and Liver Disease. Philadelphia: Saunders; 2002.

27. Swirski FK, Nahrendorf M, Etzrodt M, Wldgruber M, Corte-Retamozo V, Panizzi P, et al. Identification of splenic reservoir monocytes and their deployment to inflammatory sites. Sci 2009;325:612-6.

28. Chess L, Jiang H. Regulation of Immune Responses by $\mathrm{T}$ Cells. NEJM 2006;354:1166-76.

29. Male D, Brostoff J, Roth DB, Roitt IM. Imunologia. São Paulo: Elsevier; 2014.

30. Sawa S, Lochner M, Satoh-Talayama N, Dulauroy S, Bérard $\mathrm{M}$, Kleinschek M, et al. ROR $\gamma+$ innate lymphoid cells regulate intestinal homeostasis by integrating negative signals from the symbiotic microbiota. Nat Imm 2011;12:320-6.

31. Takayama T, Kamada N, Chinen H, Okamoto S, Kitazume MT, Chang J, et al. Imbalance of NKp44+NKp46- and NKp44-NKp46+ natural killer cells in the intestinal mucosa of patients with Crohn's disease. Gastroenterol 2010;139:88292.

32. Schroeder BO, Wu Z, Nuding S, Groscurth S, Marcinowski $\mathrm{M}$, Beiner J, et al. Reduction of disulphide bonds unmasks potent antimicrobial activity of human $\beta$-defensin 1 . Nat
2011;469:419-23.

33. Dolowschiak T, Chassin C, Mkdden SB, Fuchs TM, Weiss $\mathrm{S}$, Vandewalle A, et al. Potentiation of epithelial innate host responses by intercellular communication. PLoS Path 2010;6:1001-94.

34. Rothfuss KS, Stange EF, Herrninger KR. Extraintestinal manifestations and complications in inflammatory bowel diseases. World J Gastroenterol 2006;12(30):4819-31.

35. Rosemberg NA, Li ML, Ward R, Pritchard JK. Informativeness of genetic markers for inference of ancestry. AJHG 2002;73(6):1402-22.

36. Bastos-Rodrigues L, Pimenta JR, Pena SDJ. The Genetic Structure of human populations studied through short insertion-deletion polymorphisms. Ann Hum Gen 2006;70:658-65.

37. Seyferth G. Colonização, imigração e a questão racial no Brasil. Rev USP 2002;53:117-49.

38. Hanchard NA. Genetic susceptibility and single-nucleotide polymorphisms. Semin Fetal Neonatal Med 2005;10:283-89.

39. Young, RP, Hopkins R, Black PN, Eddy C, Wu L, Gamble $\mathrm{D}$, et al. Functional variants of antioxidant genes in smokers with COPD and in those with normal lung function. Thorax 2006;61:394-9.

40. Bica CG, Cruz IBM, Silva LLM, Toscani NV, Zettler CG, Graudenz MS. Association of manganese superoxide dismutase gene polymorphism (Ala-9Val) and breast câncer in males and females. JBPML 2007;43(3):219-25.

41. Hindorff LA, Sethupathy P, Junkins HA, Ramos EM, Mehta JP, Collins FS, et al. Potential etiologic and functional implications of genome-wide association loci for human diseases and traits. PNAS 2009;106: 9362-7.

42. Fuller SJ, Stokes KK, Skarratt BJ, Gu JS. Genetics of the P2X7 receptor and human disease. Pur Signal 2009;5:257-62.

43. Ponder A, Long MD. A clinical review of recent findings in the epidemiology of inflammatory bowel disease. Clin Epidem 2013;5:237-47. 